\title{
Kentron
}

Revue pluridisciplinaire du monde antique

19 | 2003

Le statut et l'image du corps dans la mythologie et la littérature grecques (suite et fin)

\section{Le corps du prince dans la Chronographie de Michel Psellos}

\section{Corinne Jouanno}

\section{OpenEdition}

\section{Journals}

Édition électronique

URL : https://journals.openedition.org/kentron/1863

DOI : 10.4000/kentron. 1863

ISSN : 2264-1459

Éditeur

Presses universitaires de Caen

Édition imprimée

Date de publication : 31 décembre 2003

Pagination : 205-221

ISBN : 2-84133-222-5

ISSN : 0765-0590

Référence électronique

Corinne Jouanno, «Le corps du prince dans la Chronographie de Michel Psellos », Kentron [En ligne]

19 | 2003, mis en ligne le 12 avril 2018, consulté le 13 octobre 2022. URL : http://

journals.openedition.org/kentron/1863; DOI : https://doi.org/10.4000/kentron.1863

\section{(c) $(1) \&$}

Creative Commons - Attribution - Pas d'Utilisation Commerciale - Pas de Modification 4.0 International - CC BY-NC-ND 4.0

https://creativecommons.org/licenses/by-nc-nd/4.0/ 


\section{LE CORPS DU PRINCE DANS LA CHRONOGRAPHIE DE MICHEL PSELLOS}

La Chronographie de Michel Psellos (1018-peu apr. 1078) est sans doute l'une des œuvres les plus connues de la littérature byzantine. Psellos y évoque cent années mouvementées de l'histoire de Byzance, de l'avènement de Basile II le Bulgaroctone (976-1025) à la fin du règne de Michel VII Doukas (1067-1078) ${ }^{1}$. Sous sa forme actuelle, l'ouvrage possède un caractère un peu disparate, puisqu'il est formé de deux parties composées à des époques et dans une optique différentes ${ }^{2}$ : la seconde partie, qui va du règne de Constantin X Doukas (1059-1067) à celui de Michel VII, fut en effet rédigée à la demande de ce dernier, et tient du panégyrique beaucoup plus que de l'histoire, puisqu'elle est écrite à la gloire de la dynastie des Doukas ${ }^{3}$. Mais la première partie elle-même est, à vrai dire, moins l'œuvre d'un historien que celle d'un mémorialiste, car Psellos, qui fut à la fois un écrivain prolifique et un homme d'État d'une rare longévité, secrétaire impérial, puis premier ministre et conseiller intime de plusieurs empereurs successifs, de Constantin IX Monomaque (1042-1055) à Michel VII, a été non seulement le témoin oculaire, mais souvent aussi un acteur majeur de bien des événements rapportés dans la Chronographie ${ }^{4}$. Proche du pouvoir, il nous raconte d'ailleurs moins l'histoire de Byzance que celle de ses empereurs, dont la personne occupe presque constamment le devant de la scène personne morale et personne physique, car Psellos fut à la fois un fin psychologue et un talentueux portraitiste, et c'est l'une des particularités de sa Chronographie que d'accorder au corps du prince une attention dont aucune autre ouvre contemporaine n'offre l'équivalent ${ }^{5}$.

1. Le récit de Psellos s'arrête en 1077, un an avant l'usurpation de Botaneiatès.

2. Cf. Sykoutris 1929-1930, suivi par Hussey 1935, Hunger 1978, I, 372-382, Impellizeri 1984, Kaldellis 1999.

3. Psellos, Chronographie, 7/ 4, 11 (Renauld 1967, II, 178).

4. Comme le note Hunger (1978, I, 377), la longueur des développements consacrés à chaque empereur est directement proportionnelle au rôle que Psellos joua sous le règne de tel ou tel : ainsi Constantin IX, sous lequel Psellos devint une figure politique de premier plan, bénéficie-t-il d'un développement trois fois plus long que n'importe quel autre des souverains de la Chronographie (une centaine de pages, sur les trois cent trente-neuf que compte l'édition de la CUF).

5. Au XII siècle, en revanche, l'Alexiade d'Anne Comnène, qui s'inspire de Psellos (cf. Linner 1983), offre un matériau assez comparable à celui fourni par la Chronographie. 
De fait, Psellos manque rarement de préciser l'aspect physique des souverains dont il relate l'histoire et, fait notable, ces souverains ont presque toujours belle apparence, conformément aux normes de l'idéologie impériale, qui fait de la beauté l'apanage obligé du prince ${ }^{6}:$ du moins les héros de la Chronographie sont-ils beaux en début de règne, car en général, leur corps ne tarde guère à s'altérer, comme nous allons le voir. Il arrive que la beauté de l'empereur soit évoquée en quelques lignes, voire en une simple phrase - c'est le cas pour Romain III Argyre (1028-1034), dont Psellos dit simplement: «Il avait la parole délicate et la voix pleine de majesté; c'était un héros par la stature et, par le visage, un vrai roi ${ }^{7}$. Mais on trouve aussi des portraits détaillés, ceux, par exemple, de Basile II ou de Constantin Monomaque, dont Psellos décrit la physionomie en deux ekphraseis longues d'une quarantaine de lignes ${ }^{8}$.

On est frappé d'emblée par le caractère extrêmement littéraire de ces descriptions, où abondent les souvenirs d'Homère et du roman grec: les joues de Michel VII s'ombragent d'une barbe en sa première fleur, que Psellos évoque en une formule tout droit tirée du chant XI de l'Odyssée ${ }^{9}$; Basile II est certes d'une taille un peu inférieure à la moyenne, mais offre, assis à cheval, « un spectacle en tout point incomparable», tel, au chant III de l'Iliade, Ulysse, plus petit que Ménélas, mais plus imposant que lui lorsqu'ils sont tous deux assis ${ }^{10}$; Michel IV (1034-1041) encore adolescent a «le coloris d'une fleur, l'œil brillant et les joues vermeilles», comme un héros de roman grec ${ }^{11}$; et Constantin Monomaque, avec ses cheveux blonds couleur de soleil et son teint blanc pareil au cristal, possède lui aussi un type très romanesque de

6. Ainsi Eusèbe de Césarée, père fondateur de l'orthodoxie politique byzantine, vante-t-il à plusieurs reprises la beauté de Constantin, dans la biographie qu'il nous a laissée du premier empereur chrétien : cf. Vie de Constantin, 1, 19, 2; 3, 10, 3-4; 4, 53. Dans le chapitre où il traite du basilikos logos, le rhéteur Ménandre paraît bien conseiller aux auteurs d'éloges impériaux de prêter à l'empereur « toutes

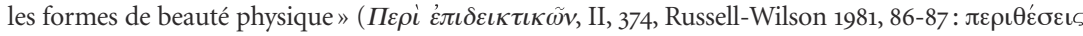

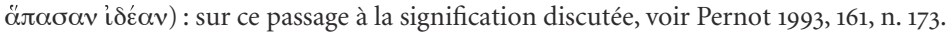

7. Psellos, Chronographie, 3,2 (Renauld 1967, I, 33).

8. Basile II : 1, 35-36 (Renauld 1967, I, 22-23); Constantin IX : 6/ 1, 125-126 (Renauld 1967, II, 30-31). Voir traduction en annexe.

9. Psellos, Chronographie, 7/ 4, 3 (Renauld 1967, II, 174); cf. Homère, Od., 11, 319-320.

10. Psellos, Chronographie, 1, 36 (Renauld 1967, I, 23) ; cf. Homère, Il., 3, 210-211: "Quand ils étaient debout, Ménélas dépassait [Ulysse] de ses larges épaules, mais quand tous deux s’asseyaient, Ulysse était plus imposant. » Notation similaire chez Anne Comnène à propos d'Alexis I ${ }^{\mathrm{er}}$ : « Il n'était certainement pas d'une haute stature, mais sa carrure était bien proportionnée à sa taille. Aussi, debout, n'excitait-il pas tellement l'admiration chez ceux qui le voyaient; mais quand il était assis sur le trône impérial et que ses yeux brillaient d'un feu terrifiant, il semblait fulgurant, tellement irrésistible rayonnait l'éclat de son visage et de toute sa personne» (Leib 1937-1946, 3, 3, 2).

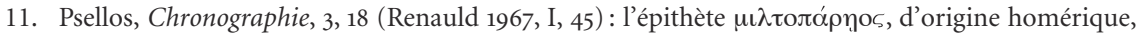
est habituellement utilisée pour parler de bateaux (cf. Homère, Il., 2, 637; Od., 9, 125). Skylitzès, plus sobre que Psellos, dit simplement que Michel IV avait très belle apparence (Thurn 1973, 390). 
beauté, que Psellos compare d'ailleurs à la beauté d'Achille ou de Nirée - rapprochement courant dans les portraits des protagonistes du roman $\operatorname{grec}^{12}$.

En accord avec les canons esthétiques de son temps, Psellos insiste sur l'harmonie des proportions, la symétrie du corps de ses héros. De Basile II, il écrit :

Sa figure tout entière, comme partant d'un centre, s'arrondissait en un cercle exact et, par un cou bien agencé et un peu long, s'ajustait au milieu des épaules. Sa poitrine n'était ni jetée en avant et comme en saillie, ni par contre ramenée en dedans et comme en retrait ; mais elle tenait une juste mesure entre les deux écarts, et les autres parties de son corps s'harmonisaient avec elle ${ }^{13}$.

L'importance accordée par Psellos à la notion de juste milieu trahit sans doute aussi la familiarité de notre auteur avec la littérature physiognomonique, qui voit dans l'équilibre des qualités le secret de la beauté parfaite et considère tout excès comme un indice de difformité morale ${ }^{14}$. L'influence de ces critères normatifs est particulièrement sensible dans le portrait de Michel VII, dont Psellos, rappelons-le, s'est fait le panégyriste :

Ses yeux sont fixes, et son sourcil n'est ni orgueilleux, ni comme soupçonneux et rabattu sur les yeux, mais libre, et avec la gravité qui convient. Sa démarche ne se traduit point par un pas précipité et comme désordonné; elle n'est pas non plus paresseuse et nonchalante : elle est telle qu'un musicien traitant des levés et posés ne pourrait que la louer; et le son de sa voix est harmonieux et bien rythmé; sa parole ne s'épanche pas en un courant qui bouillonne avec bruit, et elle n'est ni obscure ni difficile à saisir $^{15}$.

12. Psellos, Chronographie, 6/1, 126 (Renauld 1967, II, 30-31); cf. Chariton, 1, 1, 3 (Chairéas est tel que les peintres et les sculpteurs représentent Achille ou Nirée, roi de Symé, cité dans l'Iliade [2, 671674] comme «le plus beau de tous les Danaens venus sous Ilion après le Péléide sans défaut »); Héliodore, 2, 35, 1 (comparaison de Théagène à Achille). Ici encore, Skylitzès se contente d'indiquer

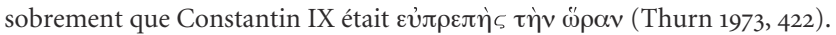

13. Psellos, Chronographie, 1, 35 (Renauld 1967, I, 22). Sur le cercle comme image de la perfection, voir Platon, Timée, $33 \mathrm{~b}$ : «Le dieu a tourné le monde en forme de sphère, où la distance du centre aux extrémités est partout égale, et [l'a fait] circulaire - forme qui, de toutes, est la plus parfaite et la plus semblable à elle-même, car il pensait que le semblable est mille fois plus beau que le dissemblable. »

14. Cf. Evans 1969, 24. Sur l'importance accordée par les physiognomonistes à la notion de juste proportion / symétrie, voir notamment Aristote, Physiognomonica, 72 : «Si les gens mal proportionnés

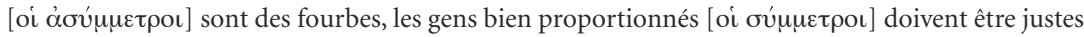
et courageux» $(S P, \mathrm{I}, 88)$.

15. Psellos, Chronographie, 7/ 4, 5 (Renauld 1967, II, 175). La démarche de Michel VII répond aux exigences formulées par Photius dans sa Lettre à Michel, prince de Bulgarie, chap. 34, PG, 102, col. 665-

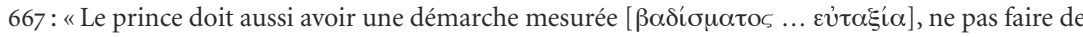
mouvements inconvenants, qui lui donnent l'air efféminé et dissolu, pas non plus de mouvements téméraires, qui lui donnent l'air brusque, agité, déséquilibré. En un mot, que tous ses gestes soient

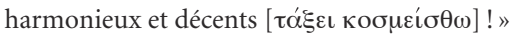


Ce portrait de Michel VII est par ailleurs en parfait accord avec ce que A. Kazhdan a nommé l'«idéal statuesque » des Byzantins ${ }^{16}$ - un idéal de comportement qui prescrit d'éviter les mouvements inutiles, les gesticulations brusques et désordonnées, pour apparaitre calme et solennel, pareil à une statue ${ }^{17}$ - idéal dont certaines descriptions de Constance II offrent un exemple précoce ${ }^{18}$ et que l'on retrouve régulièrement invoqué dans les éloges d'empereurs byzantins ${ }^{19}$. Basile II, dont Psellos souligne la prestance à cheval, est lui aussi pareil à ces «statues que les maîtres sculpteurs ont façonnées pour une telle posture », tant il apparaît comme «moulé sur sa selle ${ }^{20}$.

À la différence, pourtant, des descriptions physiques très stylisées figurant dans les divers discours panégyriques composés par Psellos en l'honneur de Constantin Monomaque, descriptions où il n'est guère question que de l'éclat du souverain, de ses cheveux d'or, de son teint de lys et de rose ${ }^{21}$, les portraits insérés dans la Chronographie témoignent parfois d'un certain effort d'individuation. Dans le long portrait de Basile II, cette tendance apparaît de façon très évidente lorsque Psellos en vient à parler du comportement physique de l'empereur, et note l'habitude qu'avait

16. Kazhdan 1991.

17. Le même idéal statuesque transparaît dans le portrait que Psellos trace d'Isaac I ${ }^{\mathrm{er}}, \mathrm{cf} .7 /$ 1, 8 (Renauld 1967, II, 87) : «Ceux [de ses soldats] qui étaient en faute, il les terrifiait d'un seul regard, et un simple froncement des sourcils le dispensait de recourir à tout châtiment corporel » ; 7 /1, 49 (Renauld 1967, II, 113) : «Un simple signe, un geste de la main, une inclinaison de la tête dans un sens ou dans l'autre, tout cela, il le jugeait suffisant pour indiquer sa volonté. »

18. Cf. Ammien Marcellin, 16, 10, 10 (Galletier \& Fontaine 1968) : «Comme s'il eût le cou pris dans un carcan, il portait son regard droit devant lui, sans tourner le visage à droite ni à gauche et, semblable à une statue, on ne le vit jamais faire un mouvement aux cahots de son char, ni cracher, ni essuyer ou frotter son visage ou son nez, ni agiter la main.»

19. Cf. Anne Comnène évoquant la patience avec laquelle son père Alexis donnait audience aux Celtes et «endurait leur bavardage insensé »: «Telle une statue travaillée au marteau, faite en bronze ou en fer battu à froid, l'empereur restait ainsi debout toute la nuit, depuis le soir souvent jusqu'au milieu de la nuit suivante [...]. Aussi bien pas un courtisan ne pouvait-il rester debout aussi longtemps que lui, mais chacun changeait alternativement de position [...]. Seul le basileus ne bronchait pas malgré une si grande fatigue» (Leib 1937-1946, 14, 4, 7).

20. Psellos, Chronographie, 1, 36 (Renauld 1967, I, 23). Comme dans le portrait de Michel VII, l'influence de la physiognomonie est très sensible dans le portrait de Basile $\mathrm{I}^{\mathrm{er}}$, notamment dans les premières lignes : «Quant à sa forme physique, elle accusait la noblesse de sa nature. Son œil, en effet, était d'un bleu clair $[\chi \alpha \rho \circ \pi$ òv $]$ et plein de feu, et son sourcil [...] bien arqué exprimait la fierté du personnage » (1,35: Renauld 1967, I, 22). Cf. Aristote, Physiognomonica, 13 (SP, I, 26, où l'œil $\chi \alpha \rho$ oлóv est

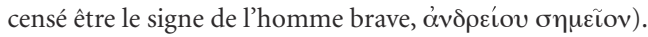

21. Cf. Psellos, Or. 1, 1. 6-7: «De l'éclat de ta beauté, tu illumines toute la terre»; 1.350-351: «N'es-tu pas d'une beauté charmante, plus que [tous] les fils des hommes?»;1. 362-366: "Avec tes cheveux dorés, tu es resplendissant, tu brilles de couleurs inimitables, tu as l'éclat d'une beauté indicible, tu possèdes un rayonnement inexprimable: n'es-tu pas tout entier soleil, n'es-tu pas plus brillant que le soleil ?»; Or. 2, 1. 471-478: "Vois quelle est la grâce de ton corps [...] : le buisson de roses de ton visage, le charme de tes yeux, la blancheur qui se répand dans la pourpre pour s'y mêler, les resplendissants rayons de ta tête!» 
Basile de rouler sa barbe entre ses doigts, «surtout quand il était enflammé de colère ", de mettre les mains sur ses hanches " avec le coude en saillie», de parler "plus à la façon d'un paysan qu'à celle d'un homme de bonne éducation", et de rire d'un "rire à éclats, avec un bouillonnement de tout son corps ${ }^{22}$ - comportement parfaitement atypique, pour ne pas dire tout à fait choquant de la part d'un empereur, et qui suggère chez Basile II une rusticité évidemment en rapport avec le mépris des lettres et des lettrés que lui a reproché Psellos quelques chapitres plus haut ${ }^{23}$.

Dans l'ensemble de la Chronographie, la place faite à la beauté des empereurs est toutefois bien moindre que celle impartie à l'évocation de leurs maladies et du délabrement physique qui en découle. Il s'agit là d'une particularité propre à Psellos, et dont on ne retrouve absolument pas l'équivalent dans les ouvrages historiques, contemporains, de Jean Skylitzès ou de Michel Attaleiatès ${ }^{24}$ - particularité d'ailleurs circonscrite à la première partie de la Chronographie, car dans la seconde partie, de caractère panégyrique, les informations médicales deviennent fort rares, et Psellos ne s'appesantit guère sur les problèmes de santé des souverains à la gloire desquels il écrit ${ }^{25}$. La place que notre auteur accorde, dans la première partie de son œuvre, aux maux physiques des empereurs, l'évidente complaisance avec laquelle il décrit leurs pathologies, sans reculer devant les précisions anatomiques les plus crues ${ }^{26}$, sont sans doute imputables à son intérêt pour la médecine - un intérêt qu'il se plaît d'ailleurs à mettre en scène dans la Chronographie ${ }^{27}$ : il s'y attarde en effet sur le rôle

22. Psellos, Chronographie, 1, 36 (Renauld 1967, I, 23). Attitude tout à fait contraire aux recommandations faites par Photius dans sa Lettre à Michel, prince de Bulgarie, chap. 36 : «Rire aux éclats, outre

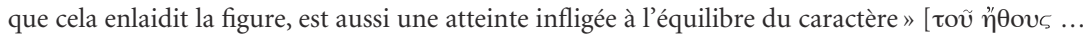

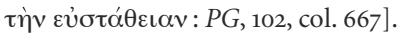

23. Psellos, Chronographie, 1, 29 (Renauld 1967, I, 18).

24. Anne Comnène, en revanche, évoque fréquemment les crises de goutte de son père $(12,3,4 ; 14,4$, 2 et $8 ; 14,5,2 ; 14,7,9 ; 15,1,1)$, et elle nous a laissé de son agonie une description très circonstanciée $(15,11,1-20)$.

25. Cf. Volk 1990, 417-418. De Constantin X, Psellos se contente de noter qu'il devint «longtemps avant sa mort la proie d'une maladie terrible» (Psellos, Chronographie, 7 / 2, 26 ; Renauld 1967, II, 151), qu’il guérit pourtant, mais que par la suite «son corps commença à dépérir » et que «peu à peu il fut proche de la mort» (Psellos, Chronographie, 7 / 2, 27; Renauld 1967, II, 151).

26. Voir par exemple le récit des derniers moments de Romain III : «Ayant fermé les yeux, il se mit à haleter d'une façon plus pressée; après quoi, soudain, de sa bouche largement ouverte se déverse une matière noirâtre coagulée ; là-dessus, ayant râlé deux à trois fois, il rend l'âme » (Psellos, Chronographie, 3, 26; Renauld 1967, I, 52) - ou encore l'évocation de la mort de Théodora : « Une maladie très grave s'empare de sa personne: en effet, sa faculté d'évacuation s'étant éteinte supprima l'appétit et se déchargea par les organes buccaux; puis, s'étant traduite en diarrhée abondante, après avoir rejeté presque tout ce qu'il y avait à l'intérieur, elle laissa l'impératrice au dernier souffle » (Psellos, Chronographie, 6/ 2, 19; Renauld 1967, II, 81).

27. Cf. Psellos, Chronographie, 7/ 1, 74-78 (Renauld 1967, II, 129-131). 
de conseiller médical qu'il joua auprès d'Isaac $I^{\text {er }}$ Comnène (1057-1059) atteint de pleurésie, se décrit en désaccord avec le médecin officiel de l'empereur sur le diagnostic à tirer du pouls du malade, et expose le différend en termes des plus techniques $^{28}$, visiblement fier d'étaler aux yeux du lecteur des connaissances médicales dont la réalité est confirmée par la présence, dans le corpus psellien, d'assez nombreux ouvrages à contenu médical, dont le plus connu est sans doute le Пóv $\mu \alpha$

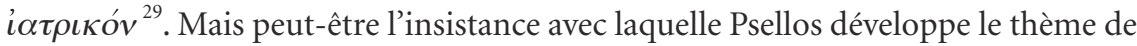
l'empereur malade tient-elle aussi à une fascination quelque peu morbide de notre auteur pour la maladie - fascination dont l'epitaphios logos de la jeune Styliané, morte d'une forme de petite vérole, offrirait un autre témoignage, s'il s'agit bien d'une œuvre authentique de Psellos, composée par le rhéteur à la mort de sa fille ${ }^{30}$. Parmi les empereurs dont la vie est racontée dans la Chronographie de Psellos, trois font figure de grands malades: Romain III Argyre, que Psellos dit atteint d'une maladie insolite et douloureuse (dont il ne précise pas la nature) ${ }^{31}$; Michel IV, qui souffrait d'une forme sévère d'épilepsie, ainsi que d'hydropisie ${ }^{32}$; Constantin Monomaque, torturé par la goutte, que Psellos omet d'ailleurs de désigner comme telle,

28. Cf. Psellos, Chronographie, $7 / 1,78$ (Renauld 1967, II, 131) : «Loin de constater un pouls en scie [ $\pi \rho i ́ o v \alpha$

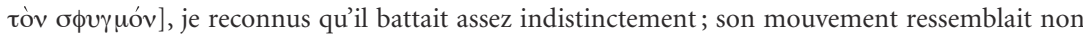

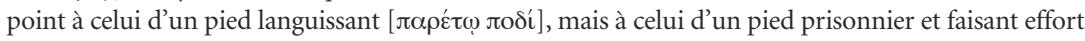

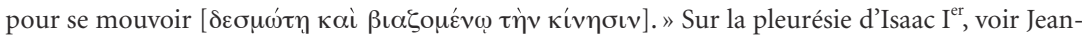
selme 1924a et Papademetriou 1996, 98-103.

29. Il y est notamment question des pulsations cardiaques (v. 61-71), de l'épilepsie (v. 785-807), de l'hydropisie (v.1128-1144), de la podagre (v. 1226-1240). Sur les écrits médicaux de Psellos, voir Volk 1990, 52-158 ( "Medizinische Monographien »). Anne Comnène, elle aussi, était férue de médecine, comme le signale le rhéteur Georges Tornikès dans l'éloge qu'il a composé à la mort de la princesse (Darrouzès 1970, 306-307).

30. Sur ce texte, voir Jouanno 1994.

31. Cf. aussi Psellos, Or. 2, 1. 246 sq. : «Malade en son corps, il devint très irascible; peu à peu, la maladie le consumait, et il fut bientôt absolument évident qu'il allait mourir : car la solidité de ses forces était détruite, son appétit s'émoussait, et le sommeil, se posant sur le bord de ses paupières, [ne] lui inspirait [plus que] des rêves éveillés; finalement, il rendit l'âme sans qu'on s'en aperçût, alors même que l'on était en train de soigner son corps. » D'après Skylitzès, Romain III aurait péri, empoisonné à petit feu par l'impératrice Zoé, éprise du jeune Michel, qu'elle souhaitait épouser (Thurn 1973, 389390). Cf. Jeanselme 1923 et Papademetriou 1996, 196-201.

32. Cf. aussi Psellos, Or. 2, l. 306 sq. : «Il tomba malade et son corps se mit à dépérir, ou plutôt fut tout

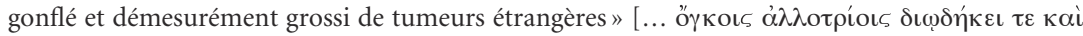

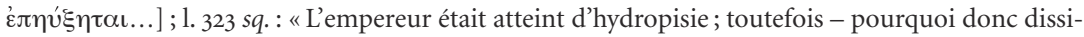
muler les faits, quand on fait œuvre d'historien, et non de panégyriste -, il souffrait aussi de crises de folie périodiques, soit qu'un mauvais démon, par malveillance, voulût abattre la partie dominante de son âme, soit qu'un suc noirâtre et terreux lui montât à la tête et obscurcît son intelligence - chose qui a coutume de se produire fréquemment. Ainsi donc, il souffrait de ces deux maux, et la vigueur de la royauté se trouvait dérobée par cette maladie »; Skylitzès, Thurn 1973, 393, 395, 405, 414-415 (brèves mentions) ; Attaleiatès, Perez Martin 2002, 7-8 (parle seulement d'épilepsie). Voir aussi Jeanselme 1924b, 230-246 et Papademetriou 1996, 68-75 et 82-89. 
sans doute en raison des connotations fâcheuses attachées à cette maladie qui frappait avec prédilection les personnes intempérantes ${ }^{33}$. Dans les trois cas, notre auteur ne se contente pas d'évoquer longuement les maux endurés par le souverain malade, mais il insiste sur la dégradation spectaculaire de son apparence physique, sur sa quasi-métamorphose. Car les trois empereurs étaient, initialement, doués d'une remarquable beauté, si l'on en croit la Chronographie.

Or Romain III qui, auparavant, «était un héros par la stature et, par le visage, un vrai roi » ${ }^{34}$, est si bien ravagé par son étrange maladie

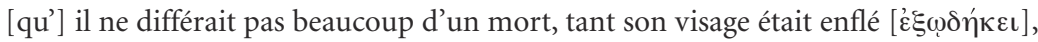
et son teint n'avait rien de plus beau que celui de ceux qui ont trois jours d'ensevelissement. [...] La plupart de ses cheveux du tour de la tête étaient tombés comme ceux d'un cadavre ${ }^{35}$;

et Psellos, qui vit de ses yeux le souverain décédé, prétend ne l'avoir " pas exactement reconnu ni au teint ni à la forme extérieure» :

C'est aux insignes seulement que j'ai compris que le mort était le basileus. C'était

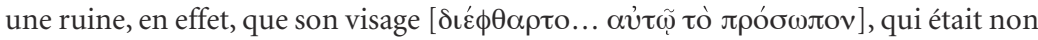
pas comme fondu, mais comme enflé [ $\omega$ s $\grave{\varepsilon} \xi \omega \delta \eta \kappa o ́ s]$; et son teint était tout changé [...] ; son poil, celui de sa tête et de sa barbe, était clairsemé de telle sorte que ses

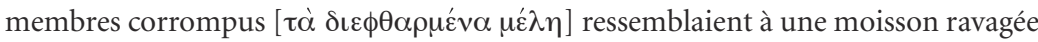
par l'incendie, dont on voit de loin la dénudation ${ }^{36}$.

On retrouve le même contraste saisissant entre l'avant et l'après dans le cas de Michel IV qui, nous l'avons vu, «avait le coloris d'une fleur, l'œil brillant et les joues vermeilles » en ses jeunes années ${ }^{37}$, mais que son hydropisie a si bien gonflé et déformé que, lorsqu'il revient victorieux de son expédition contre les Bulgares en 1041, son entrée à Constantinople ressemble moins à un triomphe qu'à un convoi funèbre :

Il allait, ballotté sur son cheval; ses doigts qui tenaient la bride ressemblaient à des doigts de géant; chacun d'eux, en effet, gardait la grosseur et la grandeur d'un bras

33. Cf. Skylitzès, Thurn 1973, 477, et Attaleiatès, Perez Martin 2002, 27, qui tous deux parlent très explicitement de podagre. Voir aussi Jeanselme 1920, 148-150 et Papademetriou 1996, 35-42. Pour l'association habituelle entre goutte et intempérance, cf. Chronographie, 2, 7 (Renauld 1967, I, 29): Constantin VIII « était dominé et par son ventre et par les plaisirs de l’amour; aussi lui était-il survenu une douleur aux articulations; mieux, il avait les pieds en si mauvais état qu'il ne pouvait pas marcher ».

34. Psellos, Chronographie, 3, 2 (Renauld 1967, I, 33).

35. Psellos, Chronographie, 3, 25 (Renauld 1967, I, 50). Détail brièvement confirmé par Skylitzès, qui parle de «maladie qui fait tomber la barbe et les cheveux » (Thurn 1973, 389).

36. Psellos, Chronographie, 4, 4 (Renauld 1967, I, 55). Psellos avait seize ans lors du décès de Romain III.

37. Psellos, Chronographie, 3,18 (Renauld 1967, I, 45). 
(dans un si grave état se trouvaient ses entrailles!), et son visage ne conservait même pas une trace de ressemblance avec ce qu'il était autrefois ${ }^{38}$.

Dans le cas de Constantin Monomaque, l'effet de contraste entre l'avant et l'après est rendu plus frappant encore par l'insistance de Psellos à vanter la beauté première de l'empereur, vrai chef-d'œuvre de la nature, dont les perfections sont énumérées en une minutieuse ekphrasis ${ }^{39}$, et par le soin que notre auteur met à souligner

de quelle vigueur et de quelle énergie fortement tendue il passa en l'état contraire, et comment, loin de conserver sa beauté intacte jusqu'au bout, tel un soleil voilé par les nuages, il montrait affaibli, à ceux qui le voyaient, l'éclat de sa nature ${ }^{40}:[\ldots]$ La durée d'une année ne s'était pas encore écoulée, voici que la nature, qui le parait pour être un objet d'admiration et de joie [...], se relâchant et s'épuisant, lui enlevait sa force et ébranlait sa beauté. Tant il y a que brusquement les principes constitutifs du corps, je veux dire les éléments essentiels, se sont désagrégés et confondus et que, tantôt aux pieds et aux creux des jointures, tantôt aux mains, ils affluaient pour, de là, inonder les muscles mêmes et les os de la région dorsale - tels des courants se précipitant ensemble sur un vaisseau auparavant solide, l'ébranlent de haut en bas. [...] Ce sont les pieds qui, les premiers, subirent le flot des fluxions [...]. Le courant, de proche en proche, se portait sur les mains, puis sur les épaules [...]. Tout membre inondé par cette fluxion terrible perdait son énergie, et les fibres et les ligaments se trouvant disjoints, les éléments de l'harmonie se sont déplacés, d'où déséquilibre [ $\alpha \rho \rho \theta \theta u i \alpha u$ ] et atonie. Et j'ai vu ses doigts si bien faits désavouer leur forme propre et, recourbés en rentrants et saillants, devenir incapables de saisir n'importe quoi; ses pieds étaient tout ramassés et repliés sur eux-mêmes; son genou enflé faisait saillie comme un coude $[\ldots]$. Il lui arrivait attaque sur attaque, ce qui rongeait le reste de ses chairs et désarticulait totalement ce qui tenait encore ${ }^{41}$.

À date ancienne, une si hideuse maladie aurait été interprétée comme un signe de la colère divine, comme un effet du jugement de Dieu : on songe à la fin horrible du persécuteur Galère qui périt rongé par un ulcère malin, et transformé dès son vivant en charogne pestilentielle ${ }^{42}$; à la «mauvaise mort» de l'hérétique Arius qui

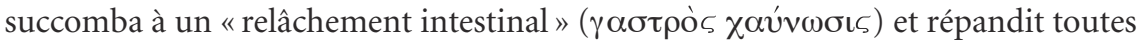
ses entrailles, comme avait fait le traître Judas ${ }^{43}$; ou bien à Héraclius qui, coupable

38. Psellos, Chronographie, 4, 50 (Renauld 1967, I, 82).

39. Psellos, Chronographie, 6/1, 125-126 (Renauld 1967, II, 30-31).

40. Psellos, Chronographie, 6/1, 124 (Renauld 1967, II, 29-30).

41. Psellos, Chronographie, 6/1,127-128 et 130 (Renauld 1967, II, 31-33).

42. Cf. Eusèbe de Césarée, Vie de Constantin, 1, 57 ; Lactance, De la mort des persécuteurs, chap. 33. Le même type de mort est attribué à Antiochos IV Épiphane dans II Macc. 9, 5-10.

43. Cf. Socrate, Hist. Eccl., I, 38, 7-9 (Hansen 1995) : s'étant rendu aux lieux d'aisance situés derrière l'agora de Constantin, Arius s'y trouve mal et laisse échapper avec ses excréments un flot de sang 
d'avoir sombré dans le monothélisme, aurait péri hydropique ${ }^{44}$. Mais Psellos écrit à une époque où le récit historique, sécularisé, ne laisse plus guère de place à ce genre d'interprétations religieuses ${ }^{45}$ - même si les reproches adressés par les ennemis de Constantin Monomaque à l'empereur malade laissent penser que, peut-être, le thème archaïque de la mauvaise mort n'avait pas perdu toute prégnance dans la mentalité populaire $^{46}$. Notre auteur, pour sa part, préfère mettre l'accent sur les souffrances, très humaines, des empereurs frappés par la maladie, et souligne volontiers le courage dont ils font preuve face aux épreuves physiques ${ }^{47}$. Il loue Michel IV d'avoir entrepris une expédition contre les Bulgares, en dépit de son triste état de santé:

Cet homme qui, la nuit, était obligé de se faire soigner et n’avait plus qu’un souffle de vie, dès que le jour paraissait, se levait soudain comme si quelqu'un lui redonnait des forces $^{48}$;

Michel IV aurait ainsi montré aux Romains «que l'ardeur ressuscite des morts et que le zèle pour les belles actions vainc la défaillance du corps » ${ }^{49}$. De même, Constantin Monomaque fait violence à la nature pour se mêler de temps en temps à la

«et le reste de ses entrailles »; le sang ayant entraîné avec lui la rate et le foie, il meurt sur place! Pour Judas, voir Actes, 1, 18: «Cet homme a acquis un domaine avec le salaire de son injustice et, tombant la tête en avant, a crevé par le milieu, et toutes ses entrailles se sont répandues. »

44. Jean Zonaras, 14, 17 (Büttner-Wobst 1897, 215-216) : "Ayant versé dans l'hérésie des Monothélites, il fut atteint d'hydropisie. Et l'on dit même que son sexe, redressé, projetait l'urine vers le haut et que, si on ne lui mettait pas une planche sur le bas-ventre, le liquide évacué, en jaillissant, lui frappait le visage. C'était là, semble-t-il, la conséquence de son union illicite avec sa nièce. » D’après Flavius Josèphe, le sinistre Hérode, condamné pour prix de ses péchés à périr de maux multiformes, aurait cumulé douleurs d'entrailles, œdème aux pieds (hydropisie), tumeur du bas-ventre et gangrène du sexe, engendrant des vers (Bell. Jud., I, 656; Ant. Jud., 17, 169 - repris par Eusèbe, Hist. Eccl., $1,8,5)$.

45. Cf. Reinsch 1994.

46. Cf. Psellos, Chronographie, 6/ 1, 110 (Renauld 1967, II, 21-22) : les ennemis de Constantin IX lui reprochent « la maladie de son corps, tantôt le qualifiant de maudit [' $\varepsilon v \alpha \gamma \tilde{\eta}]$, d'amateur de plaisirs infâmes, tantôt l'appelant ruine de la cité et corrupteur du peuple», ils improvisent à son sujet des couplets injurieux et le tournent en dérision. Anne Comnène rapporte elle aussi les moqueries dont son père, en proie à la goutte, était victime de la part des barbares, qui firent de son mal de pieds un sujet de comédie, et le représentaient, étendu sur un lit, dans l'intention de le ridiculiser $(15,1,2)$.

47. On note la même particularité chez Anne Comnène, qui loue l'endurance dont son père faisait preuve lors de ses crises de goutte, et la résignation qui le portait à dire : «Je mérite de souffrir ; c'est à juste titre que cela m'arrive à cause de la multitude de mes fautes » (14, 4, 8: Leib, 1937-1946).

48. Psellos, Chronographie, 4, 44 (Renauld 1967, I, 79).

49. Psellos, Chronographie, 4, 50 (Renauld 1967, I, 83). Cf. Or. 2, 1.346-347 : l'empereur part en expédition «sans tenir aucun compte de ce qui fait ordinairement fléchir un homme, insoucieux du double mal qui consumait son corps » [...

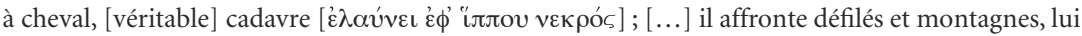
qui a besoin des bras d'autrui pour se bouger, et il est vainqueur de son adversaire. » 
foule, et mettre un terme aux rumeurs qui le donnent pour mort ${ }^{50}$, et Psellos, soulignant la patience de l'empereur, son humilité face à la maladie, y voit une attitude digne d'un «saint homme» ${ }^{51}$.

Mais, si les récits de maladie peuvent être une occasion de mettre en valeur les vertus chrétiennes de tel ou tel empereur, ils n'en attirent pas moins l'attention sur les atteintes infligées au cérémonial de la cour par la dégradation physique des souverains. Or l'empereur de Byzance, tel une icône divine, était censé manifester, par la solennité de ses apparitions publiques, la sacralité d'un pouvoir reçu de Dieu, comme le montrent bien les déclarations liminaires du Livre des cérémonies:

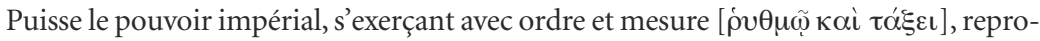

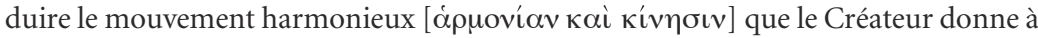

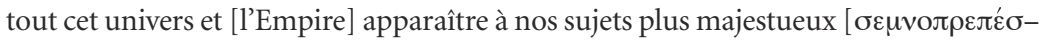
$\tau \varepsilon \rho \circ v]$ et, par là même, plus agréable et plus admirable ${ }^{52}$.

D'où la splendeur du costume impérial, et le rituel minutieusement réglé de ce que l'on qualifie parfois de véritable "liturgie aulique » ${ }^{53}$. Évoquant l'insolite et douloureuse maladie qui mine Romain III, Psellos note le cruel contraste qui se crée entre le somptueux attirail de l'empereur et son corps délabré:

Il s'habillait de vêtements splendides tout constellés d'or et il mettait sur lui toutes ses autres parures; surchargé, pour ainsi dire, de toute cette pompe avec son corps frêle, il se retournait difficilement et il se trouvait encore plus mal ${ }^{54}$.

50. Psellos, Chronographie, 6/ 1, 106 (Renauld 1967, II, 19).

51. Psellos, Chronographie, 6/1, 131 (Renauld 1967, II, 33-34). Même présentation admirative d'Isaac I ${ }^{\text {er }}$ sortant, malade, du Grand Palais: «Il n'abdiqua pas son noble caractère et il ne sortit point de sa chambre tenu par la main; mais tel qu'il était, semblable à un cyprès à la cime chevelue agitée en tous sens par le souffle des vents, il chancelait certes en s'avançant, mais il marchait quand même, quoique non maître de ses mains, et il se suffisait à lui-même » (Psellos, Chronographie, 7/1,80 ; Renauld 1967, II, 132).

52. Psellos, Chronographie (Renauld 1967, I, 2). Sur le cérémonial comme sacralisation de la personne impériale, voir Barker 1957, 101: "Ceremony played a great part in the State system of Byzantium, which made the emperor a hieratic figure moving in a solemn pageantry according to a fixed tradition"; Kazhdan 1983, 16: le rituel de la cour impériale visait à la transformation de l'empereur « into a sacral, if not sacred being».

53. Isaac $\mathrm{I}^{\mathrm{er}}$ Comnène, acclamé empereur à la faveur d'un coup de force, s'empresse d'adopter l'apparat officiel pour mieux affirmer la légitimité de sa prise de pouvoir - d'où la solennité de la réception qu'il accorde aux envoyés de son rival Michel VI : «L’empereur en personne était assis sur un trône à deux têtes, et ce trône était très élevé et plaqué d'or ; un tabouret était placé sous ses pieds et un vêtement somptueux parait son corps; il dressait haut la tête et faisait bomber sa poitrine » (Psellos, Chronographie, 7 / 1, 24 ; Renauld 1967, II, 96).

54. Psellos, Chronographie, 3, 24 (Renauld 1967, I, 50). 
Psellos insiste abondamment sur le problème posé par les apparitions publiques de Michel IV, que terrassent à l'improviste des crises d'épilepsie d'une violence extrême ${ }^{55}$, si bien que, lorsqu'il veut donner audience, des gens chargés de l'observer et de le protéger déploient devant lui de part et d'autre des étoffes de pourpre aussitôt qu'ils le voient «ébaucher quelque torsion de l'œil, ou branler la tête, ou manifester par quelque autre signe l'irruption du mal»; lorsque l'empereur sort du palais, à pied ou à cheval, il est pareillement entouré d'un cercle de gardes, prêts à le masquer aux regards importuns en cas de crise, mais qui ne parviennent pas toujours à empêcher la foule de voir le basileus choir de cheval et se débattre pitoyablement au sol ${ }^{56}$. L'état de Constantin Monomaque, si handicapé par la goutte que ses valets de chambre sont obligés de le caler et de l'appareiller avec des machines pour qu'il puisse demeurer au lit sans trop souffrir, impose lui aussi aux maîtres du cérémonial de difficiles aménagements: quand l'empereur, «impuissant à affermir sa marche et incapable de se tenir longtemps debout », veut donner audience, il faut «qu'on l'ajust [e] et qu'on l'arrang[e] à cet effet», et lorsqu'il doit sortir à cheval, "des écuyers [...] l'étayent de part et d'autre et, [...] le maintenant mutuellement comme un fardeau, le transportent là où il doit s'arrêter ${ }^{57}$. On voit ainsi le cérémonial aulique, censé offrir une image idéale du pouvoir impérial, se métamorphoser sous la plume de Psellos en une pitoyable mise en scène, qui suggère non seulement la dégradation de la personne de l'empereur, mais celle de la royauté même ${ }^{58}$.

Or un passage de la Chronographie incite précisément à lire sur le mode symbolique les successifs développements consacrés par Psellos aux maladies des souverains byzantins : il s'agit du chapitre où, évoquant les efforts entrepris par Isaac I ${ }^{\text {er }}$ Comnène pour redresser l'Empire en crise depuis la mort de Basile II, notre auteur compare l'État byzantin

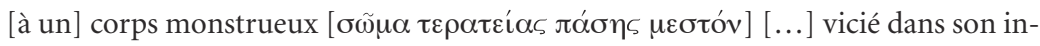

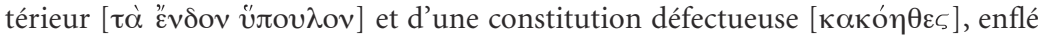

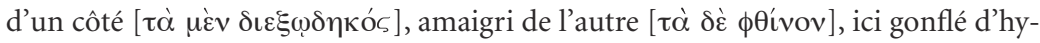

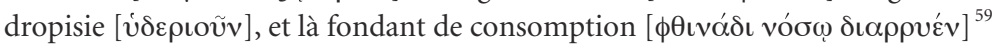

55. Cf. Psellos, Chronographie, 3, 22 (Renauld 1967, I, 48) : «Sans que survînt aucun signe prémonitoire, tout à coup il s'agitait, il roulait les yeux et s'abattait contre terre; il heurtait le sol de sa tête et pendant très longtemps, il était secoué de mouvements convulsifs. »

56. Psellos, Chronographie, 4, 18 (Renauld 1967, I, 63-64).

57. Psellos, Chronographie, 6/1, 128-130 (Renauld 1967, II, 32-33).

58. Cf. Psellos, Or. 2, 1. 323 sq. (à propos de Michel IV, miné par l'épilepsie et l'hydropisie) : «La vigueur

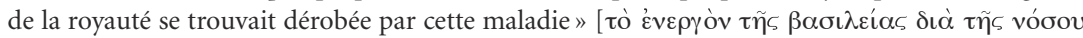

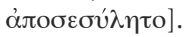

59. Psellos, Chronographie, 7/1, 51 (Renauld 1967, II, 115). Passage peut-être influencé par Aristote, Politique, 1302b, 35, dont le texte est commenté par M. Lacore dans sa contribution à ce volume : «Corps des citoyens, corps de la cité». 
- image déjà introduite dans le récit du règne de Constantin Monomaque ${ }^{60}$, mais qui se trouve ici longuement développée, dans le chapitre sur lequel devait, initialement, s'achever la Chronographie, et qui en constituait peut-être le climax ${ }^{61}$. Récapitulant à grands traits l'histoire de l'Empire depuis la mort de Basile II, époque à laquelle le corps de l'État était «florissant», Psellos file la métaphore en montrant

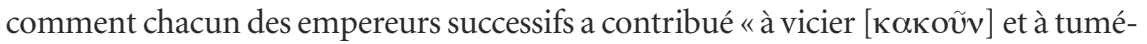

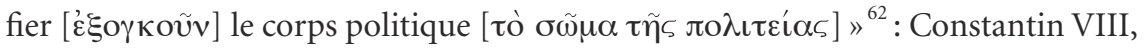

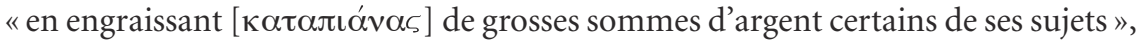

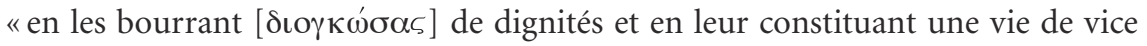

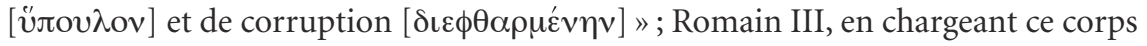

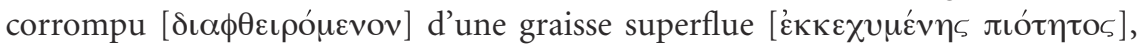
du fait de ses largesses; Michel IV en n'ayant pas la force «d'oser ôter si peu que ce fût d'embonpoint ${ }^{63}$ à ce corps habitué à se nourrir de sucs mauvais et à se gonfler

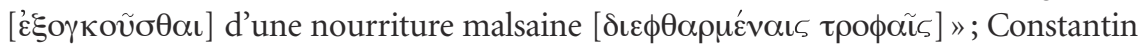

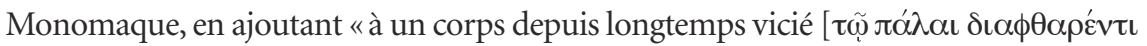
$\sigma \omega ́ \mu \alpha \tau]$ de nouvelles parties et de nouveaux membres», en introduisant dans ses entrailles «des sucs fort pernicieux», et en le faisant ainsi sortir de son état naturel - d'où la nécessité de recourir à «l'amputation, la cautérisation et la purgation », tâche à laquelle Isaac $\mathrm{I}^{\mathrm{er}}$ se serait, d'après Psellos, attelé avec une précipitation maladroite, dans son impatience à voir «le corps de l'État, devenu un corps contre nature»

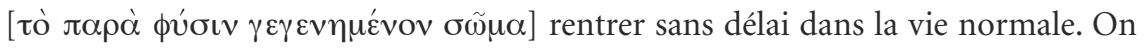
l'aura noté, les termes médicaux utilisés en ce passage sont précisément ceux qui figuraient dans les développements consacrés aux maladies des empereurs eux-mêmes,

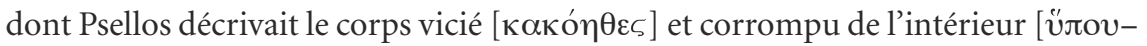

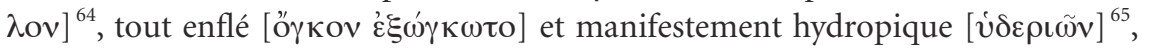

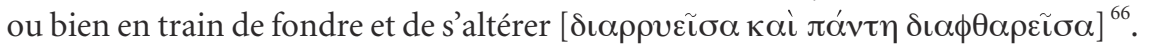

60. Cf. Psellos, Chronographie, 6/1, 48 (Renauld 1967, I, 141) : "Comme un animal vigoureux et robuste dans tout son être n'est pas tout d'un coup altéré par les premières manifestations des maladies qui l'attendent, ainsi, sous ce prince aussi, comme l'Empire ne se trouvait pas précisément alors à l'agonie, mais qu'il avait encore du souffle et de l'énergie, l'abandon à vau-l'eau ne se faisait que médiocrement sentir, jusqu'au moment où le mal, progressant peu à peu et atteignant à son comble, a tout bouleversé et confondu.»

61. Cf. Johnson 1982, 224.

62. Psellos, Chronographie, 7/ 1, 51-58 (Renauld 1967, II, 115-118).

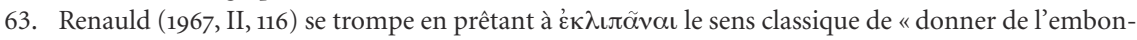
point », visiblement impropre en ce passage; pour sa part, Impellizeri (1984, II, 247) traduit par «alleggerire».

64. Psellos, Chronographie, 3, 24 (Renauld 1967, I, 49) : Romain III.

65. Psellos, Chronographie, 4, 31 (Renauld 1967, I, 71) : Michel IV.

66. Psellos, Chronographie, 6/1, 106 (Renauld 1967, II, 19) : Constantin IX. 
Ainsi le corps de l'empereur apparaît-il dans la Chronographie comme une image symbolique de l'État, lui-même conçu comme un « corps politique ${ }^{67}$ : la dégradation physique des empereurs doit par conséquent être lue comme une figure de la décadence de l'Empire - décadence que Psellos fait remonter à Constantin VIII, le frère et peu digne successeur du grand Basile II, dont le règne ouvrit pour Byzance une ère d'instabilité politique, de luttes intestines entre pouvoir civil et militaire et de constant appauvrissement, aggravé à partir des années 1025 par la poussée des périls extérieurs à toutes les frontières du royaume - Petchenègues au nord, Turcs à l'est, Normands à l'ouest ${ }^{68}$. Il est possible que la fascination morbide avec laquelle Psellos décrit, dans sa Chronographie, le dépérissement des empereurs reflète la grande pitié inspirée par le sort de l'Empire à cet homme qui a dit ailleurs son attachement à l'office impérial ${ }^{69}$; on est tenté d'y lire aussi le sentiment douloureux d'une faille entre la grandeur du métier d'empereur et l'indignité des hommes auquel incomba cette responsabilité au cours d'un siècle que l'historien Ostrogorsky qualifiait d'« ère d'épigones ${ }^{70}$.

Corinne Jouanno

Université de Caen Basse-Normandie

67. L'image est courante dans la littérature byzantine, et sa diffusion a sans doute été facilitée par la métaphore chrétienne du corps de l'Église (dont Michelle Lacore cite plusieurs exemples dans sa contribution à ce volume: «Corps des citoyens, corps de la cité») : voir, par exemple, Corippe, In laudem Justini minoris, II, 178-274 ou Anne Comnène, 1, 10, 1. Sur la personne de l'empereur comme symbole du pouvoir, voir Kazhdan 1983, 14: "The Byzantines praised the emperor as a symbol of imperial power, as an imbodiment of the everlasting Empire of the Rhomaioi. "

68. Renauld qualifie l'époque décrite par Psellos de période «la plus triste de l'histoire byzantine » (Renauld 1967, I, XVII).

69. Dans une lettre au patriarche Michel Cérulaire, Psellos oppose aux sentiments «démocratiques » de son correspondant ses propres convictions monarchiques (Ep., 207, Sathas 1876, 512) : "Quant à moi, je répète souvent le mot formulé jadis par Homère, et repris par Aristote: "Qu'un seul soit

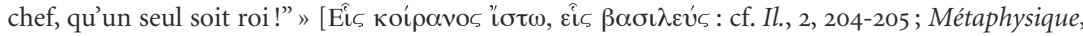

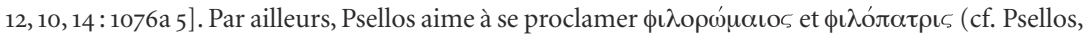
Chronographie, 6/1, 154 et 190 ; Renauld 1967, II, 46 et 64).

70. Ostrogorsky 1956,344. Kaldellis, qui voit en Psellos un esprit cynique, sorte de Machiavel avant l'heure, interprète pour sa part l'insistance avec laquelle notre auteur évoque le délabrement physique de tant d'empereurs byzantins comme une entreprise de déconstruction de la conception traditionnelle et «exaltée » de l'empereur idéal: «By exposing the all-too-human aspects of Emperors' personalities, the Chronographia deconstructs many of the myths about Imperial power» (Kaldellis 1999, $41 s q$.). 


\section{Références bibliographiques}

\section{Études}

Anastasi R. (1969), Studi sulla Chronographia di Michele Psello, Catane, Bonanno.

BArker E. (1957), Social and Political Thought in Byzantium. From Justinian I to the Last Palaeologus. Passages from Byzantine Writers and Documents, Oxford, Clarendon Press.

Evans E.C. (1969), Physiognomics in the Ancient World, Philadelphie, American Philosophical Society (Transactions of the American Philosophical Society, n.s. ; 59 / 5).

Hunger H. (1978), Die hochsprachliche profane Literatur der Byzantiner, 2 vol., Munich, C.H. Beck (Handbuch der Altertumswissenschaft ; 12.5.1-2).

Hussey J. (1935), « Michael Psellos. The Byzantine Historian », Speculum, 10, p. 81-90.

Jeanselme E. (1920), "La goutte à Byzance», Bulletin de la Société française d'histoire de la médecine, 14, p. 137-164.

Jeanselme E. (1923), «La maladie et la mort de Romain III Argyre, empereur de Byzance », Bulletin de la Société française d'histoire de la médecine, 17, p. 309-319.

Jeanselme E. (1924a), «La pleurésie du basileus Isaac Comnène. Une consultation médicale à la cour de Byzance, d'après le récit de Psellos », Bulletin de la Société française d'histoire de la médecine, 18, p. 89-97.

Jeanselme E. (1924b) «L'épilepsie sur le trône de Byzance », Bulletin de la Société française d'histoire de la médecine, 18, p. 225-274.

Johnson G.J. (1982), «Constantin VIII and Michael Psellos: Rhetoric, Reality and the Decline of Byzantium AD 1025-28 », Byzantine Studies, 9, p. 220-231.

Jouanno C. (1994), "Epitaphios logos. À sa fille Styliané, morte avant l'heure du mariage. Réflexions sur le cadavre défiguré et sur le rôle du corps dans le travail de deuil », Kentron, 10 / 2, p. 95-107.

Kaldellis A. (1999), The Argument of Psellos' Chronographia, Leyde, Brill.

KAZHDAn A. (1983), "Certain Traits of Imperial Propaganda in the Byzantine Empire from the Eighth to the Fifteenth Centuries ", in Prédication et Propagande au Moyen Âge. Islam, Byzance, Occident (Penn - Paris - Dumbarton Oaks Colloquia 3), Paris, PUF, p. 13-28.

Kazhdan A. (1991), «Body Language », in A. Kazhdan et al., Oxford Dictionary of Byzantium, Oxford, Oxford University Press, vol. I, p. 299-300.

Linner S. (1983), "Psellus' Chronographia and the Alexias. Some Textual Parallels », Byzantinische Zeitschrift, 76, p. 1-9.

Ostrogorsky G. (1956), Histoire de l'État byzantin, trad. fr. J. Gouillard, Paris, Payot.

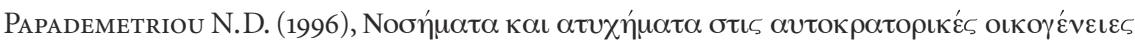

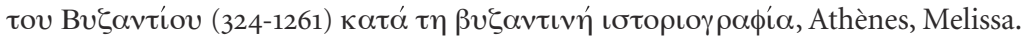

Pernot L. (1993), La Rhétorique de l'éloge dans le monde gréco-romain, Paris, Institut d'études augustiniennes (Série Antiquité; 138).

Reinsch R. (1994), «Der Tod des Kaisers », Recht-historisches Journal, 13, p. 247-270.

Renauld É. (1920), Étude de la langue et du style de Michel Psellos, Paris, Picard. 
Syкоuтris J. (1929-1930), "Zum Geschichtswerk des Psellos », Byzantinische Zeitschrift, 30, p. 61-67.

Tinnefeld F.H. (1971), Kategorien der Kaiserkritik in der byzantinischen Historiographie von Prokop bis Niketas Choniates, Munich, W. Fink.

Volk R. (1990), Der medizinische Inhalt der Schriften des Michael Psellos, Munich, Institut für Byzantinistik und neugriechische Philologie der Universität (Miscellanea Byzantina Monacensia; 32).

\section{Éditions}

Michel Psellos

- Chronographie

- É. Renauld (éd.) (1967), Paris, Les Belles Lettres (CUF), 2 vol.

- S. Impellizeri (éd.) (1984), Imperatori di Bisanzio, Milan, Mondadori, 2 vol.

- Orationes panegyricae, G. T. Dennis (éd.) (1994), Stuttgart - Leipzig, Teubner.

- Ponêma iatrikon, J.-F. Boissonade (éd.) (1829), Anecdota Graeca I, Paris, p. 175-232 (réimpr. Hildesheim, G. Olms, 1962).

- Lettres, C.N. Sathas (éd.) (1876), Bibliotheca graeca medii aevi V, Paris, Maisonneuve (réimpr. Hildesheim, 1972).

\section{Autres auteurs}

Ammien Marcellin, Histoire, t. I (1. XIV-XVI), E. Galletier, F. Fontaine (éd.) (1968), Paris, Les Belles Lettres (CUF).

Anne Comnène, Alexiade, B. Leib (éd.) (1937-1946), Paris, Les Belles Lettres (CUF), 3 vol.

Constantin Porphy rogénète, Le Livre des cérémonies, A. Vogt (éd.) (1935-1940), Paris, Les Belles Lettres (CUF), 4 vol.

Eusèbe, Vie de Constantin, F. Winkelmann (éd.) (1909), Über das Leben des Kaisers Konstantin, Berlin, Akademie Verlag - J.C. Hinrichs (Die griechischen christlichen Schrifsteller der ersten Jahrhunderte) (réimpr. 1991).

Georges et Démètrios Tornikès, Lettres et discours, J. Darrouzès (éd.) (1970), Paris, CNRS. JeAn Skylitzès, Synopsis Historiarum, I. Thurn (éd.) (1973), Berlin, De Gruyter (CFHB 5). Jean Zonaras, Annales, vol. III, T. Büttner-Wobst (éd.) (1897), Bonn, Weber (CSHB).

Ménandre Le rhéteur, D.A. Russell, N.G. Wilson (éd.) (1981), Menander Rhetor. Edited with Translation and Commentary, Oxford, Clarendon Press.

Michel Attaleiatès, Histoire, I. Perez Martin (éd.) (2002), Madrid, Consejo Superior de Investigaciones Cientificas (Nueva Roma; 15).

SCRIPTORES PHYSIOGNOMONICI GRAECI ET LATINI [abrégé en SP], R. Förster (éd.) (1893), Leipzig, Teubner, 2 vol.

Socrate, Histoire ecclésiastique, G.C. Hansen (éd.) (1995), Sokrates Kirchengeschichte, Berlin, Akademie Verlag (GCS n.f. 1). 


\section{ANNEXE}

\section{Portrait de Basile II}

\section{(Chronographie, 1, 35-36 = Renauld 1967, I, 22-23)}

"Quant à sa forme physique, elle accusait la noblesse de sa nature. Son œil, en effet, était d'un bleu clair et plein de feu, et son sourcil, qui n'était ni surplombant et sombre, ni tendu en ligne droite comme celui d'une femme mais, bien arqué, exprimait la fierté du personnage. Il n'avait pas les yeux trop enfoncés, signe de fourberie et de violence, ni trop saillants, indice de frivolité, mais ils brillaient d'un éclat viril. Sa figure tout entière, comme partant d'un centre, s'arrondissait en un cercle exact et, par un cou bien agencé et un peu long, s'ajustait au milieu des épaules. Sa poitrine n'était ni jetée en avant et comme en saillie, ni par contre ramenée en dedans et comme en retrait ; mais elle tenait une juste mesure entre les deux écarts, et les autres parties de son corps s'harmonisaient avec elle. Pour la taille, il l'avait un peu inférieure à la moyenne; mais elle était bien proportionnée aux autres parties du corps et nullement inclinée. Or donc, si on le rencontrait à pied, on pouvait certes le mettre en parallèle avec quelques personnes; mais à cheval, il offrait un spectacle de tout point incomparable. Car il était moulé sur sa selle comme le sont les statues que les maîtres sculpteurs ont façonnées pour une telle posture. Abandonnait-il les rênes à son cheval pour le précipiter en avant? Droit et ferme sur sa selle, on le voyait porté sur le terrain en pente tout comme sur le terrain à pic; et puis, contenant et arrêtant d'un coup de bride sa monture, il sautait par dessus comme s'il avait des ailes, et il conservait la même pose dans la montée et dans la descente. Dans sa vieillesse, sa barbe s'était dégarnie sous le menton; mais celle qui poussait de ses joues, le poil en était fourni et avait crû en abondance autour de son visage, si bien que, enroulée de chaque côté, elle s'achevait en cercle et qu'il semblait être partout couvert de barbe. Aussi bien la roulait-il souvent entre ses doigts, surtout quand il était enflammé de colère. Donc il faisait souvent cela, ou encore, avec le coude en saillie, il jetait ses doigts sur ses hanches. Et il parlait non pas avec volubilité, non pas en arrondissant ses phrases ou en les développant par périodes, mais en les coupant et en faisant de petites pauses, et plus à la façon d'un paysan qu'à celle d'un homme de bonne éducation. Et son rire était un rire à éclats, avec un bouillonnement de tout son corps.» 


\section{Portrait de Constantin Monomaque (Chronographie, 6 / 1, 125-126 = Renauld 1967, II, 30-31)}

«C'est une merveille de beauté que la nature, en ce prince, a donnée à la vie, la proportionnant avec tant d'art, la façonnant avec tant d'harmonie, qu'elle n'a produit, de notre temps, personne qui lui fût comparable. À cette belle proportion, elle a ajouté une vigueur fortement tendue, mettant en quelque sorte à un bel édifice de solides fondements; et cette vigueur, ce n'est pas dans la longueur des mains ni dans la grandeur des autres parties du corps qu'elle l'a renfermée ; mais elle l'a cachée, je pense, dans les profondeurs du cœur, négligeant de la révéler dans l'extérieur visible de son corps, où éclataient bien plus la beauté et l'harmonie que des proportions extraordinaires. En lui, en effet, les mains et surtout les doigts étaient de juste mesure; mais il avait de la force malgré ces proportions moyennes, et il n'était pas d'objet, si solide et si résistant fût-il, qui ne s'écrasât facilement sous l'étreinte de ses mains. Et s'il lui prenait la fantaisie de serrer le bras d'une personne, il fallait à celleci de longs jours pour se remettre. On dit encore qu'il montait à cheval dans la perfection, qu'il était le plus rapide des hommes à la course, souple, léger, absolument sans rival au pentathle. Telle était sa force, l'agilité de son corps, la rapidité de ses pieds. Sa beauté était, nous dit-on, celle d'Achille ou de Nirée; mais ces héros, la langue poétique qui, à sa fantaisie, doua leur corps de toute espèce de beauté, parvint à peine à les dépeindre ; pour Constantin, au contraire, la nature, qui le forma et le polit dans la réalité, qui pour ainsi dire le cisela et l'embellit de toute son industrie, a surpassé, par la seule puissance de son art, l'effort enchanteur de la poésie. Et lorsqu'elle eut fait chacun de ses membres en proportion avec l'ensemble de son corps, sa tête et ce qui la suit immédiatement, ses mains et ce qui les accompagne, ses jambes et ses pieds, elle répandit sur chaque partie les couleurs convenables; sa tête, elle la fit brillante comme le soleil, d'un blond ardent; et toute la poitrine et le ventre jusqu'aux pieds, ainsi que le derrière du corps, elle les a remplis de la plus pure blancheur, tant elle a bien pris ses mesures. Et certes, si quelqu'un avait voulu le considérer avec attention alors qu'il était dans la fleur de l'âge et que ses membres n'étaient pas encore flétris, c'est à la splendeur du soleil qu'il eût comparé sa tête, dont la chevelure brillait comme des rayons, c'est au cristal le plus pur et le plus diaphane qu'il eût assimilé le reste de son corps. Quant à son caractère, il était également créé pour l'harmonie, car sa langue avait une parole pleine d'urbanité, un charme s'attachait à sa conversation, et s'il venait à sourire, on voyait aussitôt les plus purs attraits des grâces. » 